\title{
Photoinduced bond oscillations in ironpentacarbonyl give delayed, synchronous bursts of carbonmonoxide release
}

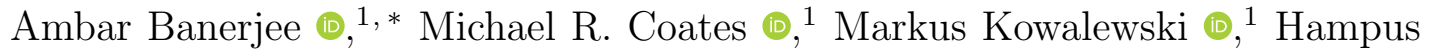

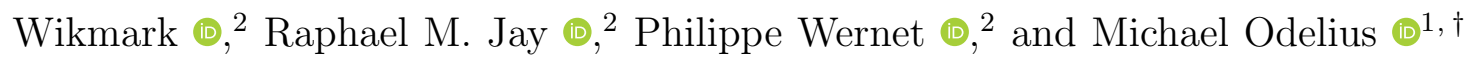 \\ ${ }^{1}$ Department of Physics, Stockholm University, \\ AlbaNova University Center, SE-106 91 Stockholm Sweden \\ ${ }^{2}$ Department of Physics and Astronomy, \\ Uppsala University, Box 516, SE-751 20 Uppsala, Sweden
}

\begin{abstract}
Early excited state dynamics in the photodissociation of transition metal carbonyls determines the chemical nature of short-lived catalytically active reaction intermediates. However, timeresolved experiments have not yet revealed mechanistic details in the sub-picosecond regime. Hence, in this study the photoexcitation of ironpentacarbonyl $\mathrm{Fe}(\mathrm{CO})_{5}$ was simulated with semi-classical excited state molecular dynamics. We find that the bright metal-to-ligand charge-transfer (MLCT) transition induces synchronous Fe-C oscillations in the trigonal bipyramidal complex leading to periodically reoccurring release of predominantly axial CO. Metaphorically the photoactivated $\mathrm{Fe}(\mathrm{CO})_{5}$ acts as a $\mathrm{CO}$ geyser, as a result of dynamics in the potential energy landscape of the axial Fe-C distances and non-adiabatic transitions between manifolds of bound MLCT and dissociative metal-centered (MC) excited states. The predominant release of axial CO ligands and delayed release of equatorial $\mathrm{CO}$ ligands are explained in a unified mechanism based on the $\sigma^{*}(\mathrm{Fe}-\mathrm{C})$ anti-bonding character of the receiving orbital in the dissociative MC states.
\end{abstract}

*ambarpchem@gmail.com

$\dagger$ odelius@fysik.su.se 


\section{INTRODUCTION}

Transition metal carbonyls have a rich legacy of well known photochemistry [1 3]. The nature of the metal carbonyl bond and its cleavage under photoexcitation is a fundamental problem in chemistry with applications in catalysis and synthesis [1]. The photochemistry of more complicated cases of transition metal carbonyls containing metal-metal bonds, opens alternative reaction pathways of energy relaxation [4. There has been a renewed interest in these compounds with the advent of ultrafast spectroscopic techniques, which provide means to precisely measure the initial photophysics and ultrafast evolution of the electronic structure [5, 6]. The choice of especially first row transition metal carbonyls for these studies is motivated by their relative simplicity in terms of electronic structure and chemistry, due to the small number of electrons and limited relativistic effects. Of these systems, ironpentacarbonyl $\mathrm{Fe}(\mathrm{CO})_{5}$ has been one of the most thoroughly investigated systems [7], in particular the processes initiated by photoinduced metal-to-ligand charge transfer (MLCT). This includes attempts to study the ultrafast molecular dynamics (MD) using UV pump probe and ionization techniques [2, 8]. Trushin and coworkers established, in their femtosecond dynamics study, the sequential dissociation of carbonmonoxide $(\mathrm{CO})$ from $\mathrm{Fe}(\mathrm{CO})_{5}$ into $\mathrm{Fe}(\mathrm{CO})_{4}$ and subsequently $\mathrm{Fe}(\mathrm{CO})_{3}$, with the first step happening within $100 \mathrm{fs}$ [8]. The generation of $\mathrm{Fe}(\mathrm{CO})_{4}$ from $\mathrm{Fe}(\mathrm{CO})_{5}$ has also been established using ultrafast electron diffraction studies, and there the gas phase dynamics is suggested to follow a singlet pathway leading to generation of $\mathrm{Fe}(\mathrm{CO})_{4}$ in an electronic singlet state [9]. Time-resolved valence and core-level photoelectron spectroscopy employing an x-ray free-electron laser has been used to establish firmly the sequential and singlet dissociation pathway of $\mathrm{Fe}(\mathrm{CO})_{5}$ in gas phase [10]. From these studies insights into the kinetics of the process and the chemical changes based on charge localization were also obtained [11]. The initial ultrafast dynamics of these systems determine how the photon energy is channeled into electronic and nuclear degrees of freedom, and eventually leads to the preferential formation of one reactive intermediate over another. The events initiating these processes are still not at all well understood primarily due to limited temporal resolution in the experiments. Hence, explicit simulations of the excited state molecular dynamics (ESMD) can yield key understanding of vibrational excitations and non-adiabatic transitions following the MLCT excitation and reaction pathways leading to the photodissociation. 
Theoretical studies have been used to gain understanding of systems similar to $\mathrm{Fe}(\mathrm{CO})_{5}$ but these also lack a detailed description of early time scale dynamics [12. The excited states of $\mathrm{Fe}(\mathrm{CO})_{5}$ have been investigated with advanced quantum chemical methods [13, 14]. With similar accuracy, the pathways of the ground state $\mathrm{Fe}(\mathrm{CO})_{5} \rightarrow \mathrm{Fe}(\mathrm{CO})_{4}+\mathrm{CO}$ reaction has been studied by Roos and co-workers [15]. Insight into the sub-picosecond dynamics in the photochemistry has direct implications for the understanding of the photochemical reactivity of these complexes, many of which have broader applications [16]. The photochemical C-H activation of transition metal carbonyls is a prime example of this [17]. The photodissociation of $\mathrm{CO}$ ligands from metal carbonyls, especially in iron complexes of which $\mathrm{Fe}(\mathrm{CO})_{5}$ is a prototypical case, is of interest to a broader audience of chemists as it has direct implication on the binding of $\mathrm{CO}$ molecules to biologically important iron complexes in solution [18]. In addition, $\mathrm{Fe}(\mathrm{CO})_{5}$ has been extensively studied lately, not only for photolytic processes, but for interesting ground state phenomena [19, 20]. These studies include the direct observation of the transition state for Berry pseudorotation [21]. The photochemistry of $\mathrm{Fe}(\mathrm{CO})_{5}$ in solvent phase is of great interest and has been studied using femtosecond X-ray (Resonant X-ray inelastic scattering - RIXS) spectroscopy [22, 23] indicating competing pathways of intersystem crossing and $\mathrm{Fe}(\mathrm{CO})_{4}$-solvent complex formation. With the advent of ab-intio excited state molecular dynamics techniques [24] these exact problems and dark areas, which could not be addressed in previous experimental studies due to limited temporal resolution, can now be investigated theoretically. This will in turn provide a challenge for experimental improvement of temporal resolution and the development of new experimental techniques altogether [24 27]. Over the past few years a wide range of problems dealing with complex photochemistry/physics of transition metal chemistry has been investigated by MD techniques which include photosensitizers and spin relaxation dynamics [28, 29]. Analogous to the $\mathrm{Fe}(\mathrm{CO})_{5}$ system, $\mathrm{Cr}(\mathrm{CO})_{6}$ has been studied with excited state MD simulations using forces from time-dependent density functional theory (TDDFT) to follow dynamics in the lowest singlet excited states $\mathrm{S}_{1}, \mathrm{~S}_{2}$ and $\mathrm{S}_{3}[30$.

In this study, we access the early stages of photochemistry/physics of $\mathrm{Fe}(\mathrm{CO})_{5}$ using excited state molecular dynamics [24] with a semi-classical treatment of non-adiabatic transitions. The $\mathrm{Fe}(\mathrm{CO})_{5}$ complex has a trigonal bipyramidal $\left(\mathrm{D}_{3 h}\right)$ ground state geometry with distinct axial and equatorial CO ligands, subject to slow exchange from Berry pseudorotation 21. After a MLCT excitation, we observe several unique features and mechanistic 
intricacies in the $\mathrm{Fe}(\mathrm{CO})_{5} \rightarrow \mathrm{Fe}(\mathrm{CO})_{4}+\mathrm{CO}$ photodissociation process. This includes an unprecedented report of synchronous bursts of $\mathrm{CO}$ at periodic intervals of $\sim 90 \mathrm{fs}$ determined by the potential energy shape of bound MLCT states. The simulations shed light on the mechanistic pathway involving photodissociation predominantly of axial $\mathrm{CO}$ and vibration relaxation involving several nuclear degrees of freedom. We also find a unique correlation between the dissociation time and angular distortion towards $\mathrm{C}_{4 v}$ symmetry in the $\mathrm{Fe}(\mathrm{CO})_{5}$ unit which indicates an alternate mechanistic pathway. Both the preferential loss of axial $\mathrm{CO}$ and the delayed dissociation at the distorted geometries, of axial and equatorial CO, can be understood from a frontier molecular orbital analysis. Population analysis reveals that the $\mathrm{Fe}-\mathrm{C}$ bond dissociation involves non-adiabatic transitions from MLCT states to metal-centered (MC) states associated with an internal electron redistribution leading to repulsive forces.

\section{RESULTS}

\section{UV spectrum and which electronic states are initially populated}

Previous studies [3, 8] suggest that the experimentally employed $267 \mathrm{~nm}$ irradiation induces a $3 e^{\prime} \rightarrow 4 e^{\prime \prime *}$ orbital transition and targets a MLCT state $\left({ }^{1} \mathrm{~A}_{2}^{\prime \prime}\right)$. Low lying electronic states and frontier orbitals of $\mathrm{Fe}(\mathrm{CO})_{5}$ are shown in Figure 1, following the nomenclature of the $\mathrm{D}_{3 h}$ point group in accordance with previous calculations [13]. For evaluation of our computational TDDFT framework (see Section V), we display experimental, as shown in SI for ref [22], and calculated UV spectra for gas phase $\mathrm{Fe}(\mathrm{CO})_{5}$ in Figure $1 \mathrm{a}$, along with the energies of various excited states computed at different levels of theory in Table S1 and in Figures S1 and S2 in the supplementary information (SI) [31].

The theoretically computed UV spectra in Figure 1 a were obtained for the equilibrium geometry and with a Wigner phase space sampling of 300 geometries, and reproduce the presence of a peak in the spectral region corresponding to the MLCT ${ }^{1} \mathrm{~A}_{2}^{\prime \prime}$ state. Apart from the consistent underestimation of all excitation energies, we notice in Table S1] in the SI [31] a close agreement between TDDFT and high level quantum chemistry, differing essentially only in the ordering of the quasi-degenerate bright ${ }^{1} \mathrm{~A}_{2}^{\prime \prime}$ and dark ${ }^{1} \mathrm{E}^{\prime}$ states, due to the different treatment of dynamical correlation, which earlier have been identified as being 
possibly involved in the excitation at $267 \mathrm{~nm}[3]$. We also investigated the excitation with different functionals and found the results to be in close agreement with cam-B3LYP, see Table S1. TDDFT and NEVPT2 give qualitatively equivalent UV-VIS spectra as shown in Figure S1, with TDDFT underestimating the excitation energies.

A multiconfiguration approach in the ESMD simulations would be preferable, but as seen in Table S1, the complete active space self-consistent field method (CASSCF) yields much too high excitation energies due to the lack of dynamical correlation, and n-electron valence state perturbation theory (NEVPT2) is computationally too demanding. Hence, we resort to TDDFT, which gives results in good agreement with earlier advanced studies [13, 14]. In Figure S2 in the SI [31] cuts in the potential energy surface (PES) of electronic states show similar shapes in TDDFT and NEVPT2, although there are differences in the relation between bound and dissociating states. Encouraged by the evaluation, we carried out excited state MD using surface-hopping in SHARC [32] based on TDDFT computations, as described in Sections $\mathrm{VB}$ and $\mathrm{VC}$.

\section{Excited State Molecular Dynamics}

Excited state MD simulations were carried out on the 116 initial conditions from the Wigner sampling, that correspond to excitation of the $\mathrm{S}_{6}$ state, involved in the bright MLCT ${ }^{1} \mathrm{~A}_{1}^{\prime} \rightarrow{ }^{1} \mathrm{~A}_{2}^{\prime \prime}$ transition. Population dynamics, non-adiabatic transitions and geometric coordinates were analyzed for the resulting trajectories in order to derive a detailed insight into the early events of the photodissociation. Out of the 116 trajectories, 110 trajectories showed single Fe-C bond dissociation as seen in Figure S3 in the SI [31]. This statistically corroborates the experimental finding that indeed $\mathrm{Fe}(\mathrm{CO})_{5}$ initially yields a $\mathrm{Fe}(\mathrm{CO})_{4}$ fragment as suggested by Trushin and coworkers [8]. The double dissociation trajectories and trajectories which do not undergo any dissociation were excluded from further analysis due to insufficient statistics. Secondly, and very interestingly, we found that for events of single bond dissociation, 94 trajectories (85\%) undergo release of an axial CO ligand and the remaining 16 trajectories exhibit release of an equatorial CO ligand (15\%). From a careful inspection of individual trajectories, we introduce a criterion for dissociation, based on a running averaging over the $\mathrm{Fe}-\mathrm{C}$ distance of the released $\mathrm{CO}$ group (see examples in Figure S4). The running average quenches oscillations in the Fe-C distance, which allows 
us to define a unique dissociation time $\left(\tau_{\text {dissoc }}\right)$ for each trajectory, based on a threshold of $2.5 \AA$. The value of $2.5 \AA$ is the lowest Fe-C distance being crossed only once in each of the 110 trajectories. The observations of bond oscillations and preferred axial CO release immediately raise a few questions. What is the mechanism for the major pathway of axial dissociation in terms of the states involved? Why is axial dissociation a preferred path? The fact that we have a minority pathway of equatorial dissociation, points to an alternate mechanism. How is that different from axial dissociation?

\section{Major channel - Release of axial CO ligands}

Photoexcited $\mathrm{Fe}(\mathrm{CO})_{5}$ exhibits multidimensional dynamics, but we highlight the most important coordinates to give a mechanistic picture of the majority pathway. Analysis of the trajectories having axial CO release indicates that the dissociation process (see Figure 2 a) involves

i) a prominent elongation of the $\mathrm{Fe}-\mathrm{C}$ bonds,

ii) followed by preferential elongation of an axial $\mathrm{Fe}-\mathrm{C}$ bonds, and

iii) angle distortion from $\mathrm{D}_{3 h}$ towards $\mathrm{C}_{4 v}$ symmetry.

The initiating MLCT excitation induces vibrations in all the $\mathrm{Fe}-\mathrm{C}$ bonds as seen in Figure S5 in the SI 31. The preferential release of axial CO ligands, and the correlated vibrations in the axial Fe-C bonds $\left(\mathrm{R}_{i}(\mathrm{ax}) ; i=1,2\right)$ motivated us to follow the motion in the reduced dimension of the $\mathrm{R}_{12}(\mathrm{ax})$ coordinate, defined as the distance between the two axial carbon atoms, as indicated in Figure 2a. The distribution of $\mathrm{R}_{12}(\mathrm{ax})$ is plotted as a function of time after the excitation in Figure $2 \mathbf{b}$, in which we can distinguish the dissociating molecules from the motion in those remaining intact. We observe a prominent synchronous oscillation, corresponding to a breathing mode, with an amplitude of $\sim 0.3 \AA$. At regular intervals, there are bursts of dissociation of CO. The rate of dissociation as depicted in Figure 2 $c$, which we measure based on the dissociation criterion of the running average of $\mathrm{Fe}-\mathrm{C}$ distance crossing the $2.5 \AA$ mark (see Figure S4), reveals three peak maxima around 50, 140 and 225 fs, which indicates that the release of $\mathrm{CO}$ is synchronous, with a period of $\sim 90 \mathrm{fs}$.

We notice the similarity with non-adiabatical dynamics in neutral and ionic surfaces leading to periodically occurring dissociation in simple diatomics, like NaI [33, but to the best 
of our knowledge this phenomenon has not been previously reported for photodissociation in complex systems like metal carbonyls.

\section{Energetics and Electronic states}

Having characterized the major mechanistic pathway which predominantly leads to dissociation of axial Fe-C bonds, we proceed with analyzing energetics and electronic aspects; and to be more precise how do the energies of the different electronic states change along the reaction coordinate and in which state or group of states does the dissociation happen. For this purpose, we performed rigid two dimensional (2D) PES scans of the $\mathrm{R}_{1}(\mathrm{ax})$ and $\mathrm{R}_{2}(\mathrm{ax})$ distances for the lowest ten electronic states. The adiabatic states can be partitioned into one class of bound excited states $S_{5}$ to $S_{9}$, represented by the PES of $S_{6}$ in Figure $2 d$, and another class of dissociative excited states $\mathrm{S}_{1}$ to $\mathrm{S}_{4}$, represented by the PES of $\mathrm{S}_{3}$ in Figure 2e (for all states $\mathrm{S}_{2}$ to $\mathrm{S}_{9}$ see Figure $\mathrm{S6}$ in the SI [31]). Analyzing the character at Fe-C $>2.2 \AA$ of the adiabatic states $\mathrm{S}_{1}$ to $\mathrm{S}_{9}$, we notice that in the diabatic picture (see Figure S7 in the SI [31]), they are related to bound states with MLCT character, involving excitations into the $4 e^{\prime \prime *}$ orbitals in Figure 1 $\mathbf{c}$, and four dissociative states with MC character, arising from the four $\mathrm{d}-\mathrm{d}$ excitations from the $9 e^{\prime \prime}$ and $3 e^{\prime}$ orbitals into the $14 a_{1}^{\prime *}$ orbital. To relate the molecular dynamics to the population dynamics, the 2D PES of each state was the overlayed with a scatter plot of the evolution in that state in the 94 axially dissociating trajectories. Figure $2 \mathrm{~d}$ shows how the dynamics in $\mathrm{S}_{6}$ is confined in the bound potential and tend to involve symmetric distortions, whereas in the $\mathrm{S}_{3}$ state, which has a channel of dissociation along each $\mathrm{R}_{i}(\mathrm{ax})$ distance, the trajectories bifurcate into release of either of the two axial CO ligands. The observation of dissociation in the lowest electronic states corroborates the findings of earlier studies [3, 8], which suggested that dissociation happens after non-radiative decay into dark MC states. The transition from the MLCT to a MC state is associated with a transfer of an electron from a ligand orbital $\left(4 e^{\prime \prime *}\right)$ to a metal centered orbital $\left(14 a_{1}^{*}\right)$, with a particular anti-bonding overlap symmetry between the Fe and the two axial CO groups, is what effectuates the dissociation process. This concept of the electron moving to an MO having a particular orbital overlap symmetry forms an essential part of the photo-dissociation dynamics.

In an attempt to generalize the insight from analysis of trajectories with release of an 
axial CO ligand, we followed the energies of electronic states along the ESMD trajectories. Dynamically averaged potential energy cuts along the $\mathrm{Fe}-\mathrm{C}$ coordinate, corresponding to the dissociating $\mathrm{CO}$, are derived as the energies for each adiabatic state $\mathrm{S}_{i}$ averaged over the whole set of single dissociation trajectories and shown in Figure $3 \mathbf{a}$. The averaged potentials deviate from the rigid scan in Figure $\mathbf{S 2} \mathbf{a}$, since dispersion due to distortions along other degrees of freedom is also sampled. In this trajectory based information, we clearly see two categories of excited states. Thus based on Figure $3 \mathrm{a}$, we confirm that $\mathrm{S}_{5}-\mathrm{S}_{9}$ are nondissociative adiabatic states and $\mathrm{S}_{1}-\mathrm{S}_{4}$ are dissociative adiabatic states, in agreement with the analysis of the associated rigid scans shown in Figure $2 \mathrm{~d}$,e and Figures $\mathrm{S2}$ and $[\mathrm{S} 6$ in the SI [31]. For dissociated geometries $(\mathrm{Fe}-\mathrm{C}>2.2 \AA) \mathrm{S}_{5}-\mathrm{S}_{9}$ have MLCT character and $\mathrm{S}_{1}-\mathrm{S}_{4}$ have MC character. As seen in a diabatic picture in Figure S7 in the SI [31, the MLCT and $\mathrm{MC}$ states cross in the Franck-Condon region, which implies that the characters of the adiabatic states change.

The excitation puts the system in a manifold of non-dissociative states which results in correlated vibration of the two axial Fe-C bonds giving rise to a synchronous oscillation of $\mathrm{R}_{12}(\mathrm{ax})$ having a periodicity of roughly 90 fs. This synchronous oscillation in turn induces a periodic crossing of the point where non-adiabatic transition to the dissociative surface can happen. We can conceptualize this phenomenon as periodic leakage of the wavepacket from a dissociative to a non-dissociative potential happening with the same periodicity as that of the $R_{12}(\operatorname{ax})$ oscillation in the non-dissociative surface, see Scheme 1, Thus this periodic leakage of the wavepacket from MLCT to MC (non-dissociative to dissociative) gives the period bursts of axial CO release.

\section{Population dynamics and kinetic modelling}

To understand the underlying electronic mechanisms for the modes of dissociation and synchronous oscillation summarized in Figure 2 and Scheme 1, we extracted the population dynamics from the simulations and performed kinetic modelling [34 36]. Since most of the trajectories exhibit early dissociation, the temporal analysis of electronic population in the different adiabatic states has been done from 0 to 300 fs. Notice that in about $20 \%$ cases the trajectory terminates before 300 fs following Fe-CO dissociation, due to the inability of the TDDFT framework to describe the $\mathrm{Fe}(\mathrm{CO})_{4}$ species (see Figure $3 \mathbf{b}$ ). This is not a 
severe limitation, since the event of interest has then already been sampled. In Figure $3 \mathbf{b}$, we present an analysis of the population dynamics summed into bound and dissociative adiabatic states which gives a clear insight into the dissociation. The population of all the states as a function of time as presented in Figure S8 in the SI [31]. Since the adiabatic states, analyzed in Figure $3 \mathbf{b}$, are clearly separable into the MLCT and MC states for Fe-C $>2.2 \AA$, this corroborates the earlier experimental findings that photodissociation happens due to transfer from MLCT to MC states [3, 8].

The time spent in a state averaged over the trajectories can be thought of as a measure of the lifetime of the state and they are listed in Table S2 in the SI 31. Furthermore, looking at the number of jumps in the surface hopping matrix in Table S3 in the SI [31, we notice that hops predominantly occur to adjacent adiabatic states i.e. from $\mathrm{S}_{n}$ to $\mathrm{S}_{n \pm 1}$, even though the surface-hopping algorithm is not restricted to these transitions. Based on this we did kinetic modelling (see Figure S9 and discussion in the SI [31] for details) for the population dynamics. The fitted curves, of electronic population are overlayed with the simulated data as shown in Figure S8. The area under fitted curves were taken as a measure of lifetime of different states (since due to the presence of back reactions exponential fits was not an option) and was found to match well with the lifetime data from the simulation (see Figure S2). This also establishes that the dynamics can be accurately described in a simple model, in which hopping from one state predominantly happens to the adjacent adiabatic states.

To create a more firm link between the population analysis and the nuclear dynamics, we further analyzed the trajectories of axial CO release. Of these 94 trajectories with oscillations and bursts of release of axial CO ligands seen in Figure $2 \mathbf{b}$, those dissociating in the first and second burst were analyzed separately. To understand how the non-adiabatic transition involving population transfer from the non-dissociative states to the dissociative states is related to nuclear motion, we introduce a time stamp at the crest of the $R_{12}(a x)$ oscillations. Then the population dynamics for the first burst and the second burst of axial CO release are sampled relative to the time stamps presented in Figure 3c. For trajectories which dissociate in the second burst, we see that there is a larger spread in time for the population transfer from the bound states to the dissociative states, in comparison to the population dynamics in the first burst. Because of the separation into a first and second bursts, we also see in the second burst an initial lag period before the increase in population transfer. 
This phenomenon of delayed population dynamics followed by sudden dissociation/cleavage of a bond, or ballistic dynamics, has been shown earlier for photolytic ring opening of dihydroazulene by Abedi and coworkers [37]. In their case, ballistic dynamics was proposed based on the existence of a similar profile of population dynamics involving a bound $\mathrm{S}_{2}$ state, and a dissociative $\mathrm{S}_{1}$ state. This indicates that the photodissociation of $\mathrm{Fe}(\mathrm{CO})_{5}$ can be considered ballistic, since the population transfer from an initially excited MLCT to dissociative $\mathrm{MC}$ states is moderated by $\mathrm{Fe}-\mathrm{C}$ bond length oscillations in bound states.

\section{Minority channels: Equatorial dissociation and Berry pseudorotation}

As pointed out earlier, there is a $15 \%$ release of equatorial CO ligands. This warrants an investigation into the existence of a minority mechanistic route, parallel to the major axial dissociation mechanism discussed in Figure 2. In the trajectories undergoing dissociation of an equatorial Fe-C bond, we observe that the initial dynamics starting from the $\mathrm{D}_{3 h}$ ground state geometry in the Franck-Condon region involves closing of the axial C-Fe-C angle and opening up of one of the equatorial C-Fe-C angles, similar to the notion of Berry pseudorotation [38].

The associated transformation from $\mathrm{D}_{3 h}$ to $\mathrm{C}_{4 v}$ symmetry can in general be quantified using an angular difference $\left(\Theta=\Theta_{1}-\Theta_{2}\right)$ between the largest two C-Fe-C angles $\left(\Theta_{1}\right.$ and $\Theta_{2}$ respectively) involving four unique $\mathrm{Fe}-\mathrm{C}$ bonds in the complex, as depicted in Figure 4 a. For the ground state $D_{3 h}$ geometry the measure of $\Theta$ is $60^{\circ}$, and the more $\Theta$ deviates from $60^{\circ}$, the more the structure has moved towards $\mathrm{C}_{4 v}$ symmetry, which corresponds to the transition state geometry that we see in pseudorotation. The pseudorotation leads to a loss of identity of equatorial and axial CO ligands. To get an idea of the degree of angular distortion that the system has undergone before dissociation, we measured the smallest value of the angular parameter $\Theta$ that is attained in each trajectory before release of a CO moiety, i.e. reaching $\tau_{d i s s o c}$. In Figure $4 \mathbf{b}$, we made a scatter plot of this smallest value of $\Theta$ with respect to $\tau_{\text {dissoc }}$. To investigate the sensitivity to the initial geometry, we also plot the value of $\Theta$ in the Wigner sampling as a function of the dissociation time. We clearly see that the initial distribution for $\Theta$ has no effect on the time of dissociation, whereas the degree of maximum distortion, i.e. the minimum value $\Theta$ attain before dissociation, is directly proportional to the dissociation time. In other words, those trajectories which 
dissociate later have time to undergo angular distortion. From the previous analysis of bound and dissociative adiabatic states, we can conclude that angular distortion modes are also vibrationally excited in the bound states. Hence, in the diabatic picture, the Fe-C bond oscillation and initiated pseudorotation occur in MLCT states.

At the ground state $D_{3 h}$ geometry, the $S_{5}$ and $S_{4}$ states form a degenerate pair of ${ }^{1} E^{\prime}$ symmetry. However, this degeneracy is lifted along $e^{\prime}$ vibrational modes, like the concerted closing (opening) of the axial (equatorial) C-Fe-C angles. Hence, this corresponds to a JahnTeller distortion mode. Geometry optimization of $\mathrm{Fe}(\mathrm{CO})_{5}$ in the $\mathrm{S}_{5}$ state leads to a close to square pyramidal geometry with an axial C-Fe-C angle reduced to $165^{\circ}$ and equatorial CFe-C angle opened to $140^{\circ}$. In Figure 4c, we depict the frontier molecular orbitals, in which the excited electron resides in the dissociative MC states, for the ground state geometry $\left(\mathrm{D}_{3 h}\right)$ and the $\mathrm{S}_{5}$ optimized geometry (nearly $\mathrm{C}_{4 v}$ symmetry), because the $\mathrm{S}_{5}$ state forms the lowest of the non-dissociative states.

We observed that the MO coefficients and the anti-bonding overlap are distributed over four $\mathrm{CO}$ moieties near $\mathrm{C}_{4}$ geometry, where as it is localized on the two axial COs at the ground state $\left(\mathrm{D}_{3 h}\right)$ geometry. The anti-bonding overlap, which is directly linked to the dissociation of the Fe-C bond, as discussed in detail in the next section, indicates predominantly axial dissociation for $\mathrm{D}_{3 h}$, but equal probability of axial and equatorial dissociation for $\mathrm{C}_{4 v}$. This geometry dependent anti-bonding character of the metal centered orbital ( $14 a_{1}^{\prime *}$ in $\mathrm{D}_{3 h}$ symmetry), receiving the excited electron in the dissociative MC states, explains the majority and minority mechanisms for photodissociation on a common basis. This unified mechanism of the photodissociation explains the time dependence in the relative release of axial and equatorial CO ligands seen in Figure 4c.

\section{DISCUSSION}

From this theoretical study of the mechanism of the photodissociation of ironpentacarbonyl, we can reveal a mechanistic pathway closely related to conceptual ideas in previous studies [2, 8]. However, instead of non-adiabatic transitions between states with forces acting in different direction, as suggested in Ref. 8, we observe oscillations of a Fe-C breathing mode in the MLCT state yielding regular bursts of CO release after non-adiabatic transitions to MC states. The initial fraction of dissociation is dominated by axial CO ligands, 
but due to excitation of a pseudorotation mode equatorial and axial dissociation become equivalent. The Fe-C oscillations are associated with variations in the relative energies of the MLCT and ground states, which with sufficient time resolution could be detected experimentally [22, 39].

As discussed earlier in detail from different points of view, the photodissociation happens by a transfer from non-dissociative to dissociative adiabatic states, which are associated with diabatic MLCT and MC states at long Fe-C distances. General chemists may find the orbital picture and arguments based on overlap symmetry of orbital more appealing than the state picture. Even in the case of angular distortion from $\mathrm{D}_{3 h}$ to near $\mathrm{C}_{4 v}$ geometries the same orbital overlap argument holds, which we have already discussed earlier. For trajectories which undergo pseudorotation like distortion, the frontier molecular orbital accepting an electron in the dissociative MC states at the $\mathrm{S}_{5}$ optimized geometry is shown in Figure 4c lower panel. Its is also interesting to see that the Fe-CO scan for both axial and equatorial $\mathrm{CO}$ from the $\mathrm{S}_{5}$ optimized geometry is conceptually similar to the scan from $\mathrm{D}_{3 h}$ geometry(see Figure $\mathrm{S} 10$ in SI 31]). We see that both the axial and equatorial CO photodissociation happens in the four lowest lying MC states.

On a conceptual level, we also want to mention analogue to general reaction chemistry. The overlap arguments in Figure 4c have a known parallel to ground state reactivity. The nuclear motion involved in the photo-induced release of axial CO, as shown in Figure $2 \mathbf{a}$, there is an uncanny similarity to that of a $\mathrm{S}_{\mathrm{N}} 2$ reaction. The ground state geometry of $\mathrm{Fe}(\mathrm{CO})_{5}$ has a $\mathrm{D}_{3 h}$ geometry which is similar to a $\mathrm{S}_{\mathrm{N}} 2$ transition state. Similarly on the dissociative excited state surface for the axial Fe-C, this $\mathrm{D}_{3 h}$ geometry is a saddle point (see Figure S11 in the SI [31]), with the dissociative PES actually resembling half of the ground state $\mathrm{PES}$ of a classic $\mathrm{S}_{\mathrm{N}} 2$ reaction. Thus, the $\mathrm{Fe}(\mathrm{CO})_{5}$ molecule in a $\mathrm{MC}$ state at $\mathrm{D}_{3 h}$ geometry will follow the $\mathrm{S}_{\mathrm{N}} 2$-like nuclear motion down hill on a dissociative PES. From an orbital overlap point of view, the $\mathrm{S}_{\mathrm{N}} 2$ reaction happens as a consequence of populating a antibonding orbital as shown in Figure S11 in the SI [31]. In the MC state for $\mathrm{Fe}(\mathrm{CO})_{5}$, the iron $3 \mathrm{~d}_{z^{2}}$ orbital and the $\sigma^{*}$ orbitals of the CO groups are in antibonding combination, which are localized to axial COs for $\mathrm{D}_{3 h}$ geometry and delocalized on all four CO for near $\mathrm{C}_{4 v}$ geometries as discussed earlier, see Figure 4c and Figure S12, This orbital overlap symmetry and population of antibonding orbitals (similar to the frontier molecular orbital situation in $\mathrm{S}_{\mathrm{N}} 2$ reaction, see Figure $\left.\mathrm{S} 11\right)$, is what drives the release of axial $\mathrm{CO}$ from $\mathrm{Fe}(\mathrm{CO})_{5}$ following 
similar nuclear dynamics as the $\mathrm{S}_{\mathrm{N}} 2$ reaction.

\section{CONCLUSION}

Our ab initio excited state MD simulations give a detailed mechanistic insight into the photodissociation of $\mathrm{Fe}(\mathrm{CO})_{5}$, which makes for a classic text book example of transition metal chemistry. To sum up our results are highlighted below in a pointwise manner:

i) We found preferential axial $\mathrm{CO}$ release and only a minor fraction of equatorial $\mathrm{CO}$ release. This finding is a major step in the correct prediction of photodissociation of $\mathrm{Fe}(\mathrm{CO})_{5}$ which can be addressed by different experimental techniques.

ii) The population dynamics of states of different character has been described in detail, which can be directly accessed in spectroscopic studies.

iii) We have unearthed an unprecedented phenomenon, wherein CO dissociation happens in periodic bursts, as a consequence of periodic transition of the system from MLCT to MC states mediated by non-adiabatic coupling between them.

iv) We have looked into the frontier molecular orbital character of the excited states, especially the dissociative states for $\mathrm{Fe}(\mathrm{CO})_{5}$ and presented justification for the selective dissociation based upon antibonding orbital overlap symmetry. As schematically depicted in Scheme 1 the dissociation happens in reaching the MC states and the orbital involved in these states as shown in Figure 4c clearly presents the picture how for $D_{3 h}$ geometries the dissociation is preferentially axial and for near $\mathrm{C}_{4 v}$ geometries there is equal probability of dissociation of axial or equatorial Fe-C bonds.

Hence, we conclude that both the majority channel of axial CO ligand release and the minority channel of delayed release of equatorial CO ligands can be understood in a unified mechanism of photodissociation, based on orbital anit-bonding character, with conceptual similarities with the ground state $\mathrm{S}_{\mathrm{N}} 2$ reaction. The points discussed just above have implications for future experiments which would i) verify whether axial or equatorial CO leaves during photodissociation, ii) gather spectroscopic signals of oscillation in the non-dissociative states and periodic photo-ejection, or in bursts ejection, of CO. The non-adiabatic couplings between bound MLCT states and dissociative MC states depend on their relative energies, 
and will hence vary with choices of transition metal and ligands. Hence, further simulations of different carbonyl complexes are required to establish general insights.

\section{ACKNOWLEDGMENTS}

M.O. acknowledges funding from the European Union's Horizon 2020 research and innovation programme under the Marie Skłodowska-Curie grant agreement No 860553. A.B. and M.O. acknowledge funding from the Carl Tryggers Foundation (contract CTS18:285), and M.K. acknowledges funding from the Swedish Research Council (grant agreement no. 2018-05346). The calculations were partially enabled by resources provided by the Swedish National Infrastructure for Computing (SNIC) at the Swedish National Supercomputer Center (NSC), the High Performance Computer Center North (HPC2N), and Chalmers Centre for Computational Science and Engineering (C3SE) partially funded by the Swedish Research Council through grant agreement no. 2018-05973.

Competing Interests The authors declare no competing financial or non-financial interests.

Author contributions The project was designed and led by A.B and M.O in dialogue with Ph.W and M.K. Simulations were performed and analyzed by A.B. and M.C. Kinetic modelling was performed by H.W. and R.J. The manuscript was written by A.B., M.C., and M.O. All authors contributed to the manuscript.

Correspondence Correspondence and requests for materials should be addressed to A.B. and M.O.

\section{METHODS}

\section{A. Ground state geometry and Wigner distribution}

Based on the CASPT2(12,12)/TZVP optimized geometry for $\mathrm{Fe}(\mathrm{CO})_{5}$ as mentioned by Wernet et al. [22], vibrational modes were obtained at the DFT/B3LYP/cc-pVDZ level of theory using the MOLPRO package, interfaced to SHARC has been used [32]. We also checked the variation of geometry at DFT level of theory and found that cam-B3LYP which is used for ESMD in this study and TPSSH which is often the best functional for transition metal system predicts very similar geometry to the CASPT2(12,12) geometry. 
All these methods predicted the Fe- $\mathrm{C}(\mathrm{ax})=1.80 \AA$ and $\mathrm{Fe}-\mathrm{C}(\mathrm{eq})=1.81 \AA$. Density functional theory (DFT) is known to accurately predict the harmonic frequencies for single reference systems. Using the vibrational normal modes, a Wigner sampling of 300 points in phase space was created. The simulated UV spectra was created from the discrete transitions in the TDDFT and NEVPT2 computations at the equilibrium geometry and from the 300 points of the Wigner sampling, followed by application of Gaussian convolution of $0.8 \mathrm{eV}$ full-width half-maximum.

\section{B. SHARC simulations}

For the ab-initio excited state dynamics using surface-hopping, SHARC version 2.1 has been used [24, 32, 40]. Using the the Wigner distribution an absorption spectrum was calculated as formed by 7 singlet states. We avoided a larger number of excited states to enhance the fraction of trajectories initiated in $\mathrm{S}_{6}$ stats, which primarily corresponds to the energy range of the commonly used experimental UV pulse. Inclusion of more higher lying states would invariably put a larger chunk of trajectories to the region at lower wavelengths and would not correspond to the experimental setup.

Further details on the ORCA calculations are discussed in the Quantum Chemistry section VC. Of the 300 initial conditions in the Wigner sampling, we found that 116 trajectories could be excited into, or in other words landed up in, the $\mathrm{S}_{6}$ state (having ${ }^{1} \mathrm{~A}_{2}^{\prime \prime}$ state character), following the protocol suggested in Ref. [41] as implemented in SHARC [24, 32].

These 116 trajectories were propagated from the $\mathrm{S}_{6}$ state, now considering 10 singlet states. The choice of conducting the dynamics in only the singlet manifold is motivated from earlier experimental work which suggest a solely singlet pathway in gas phase [9, 10]. Since the inclusion of triplet states would significantly increase the effort, we limited our investigation of the early stages of photodissociation in $\mathrm{Fe}(\mathrm{CO})_{5}$ to include singlet states. The trajectories were run for up to 600 fs but many trajectories terminated much earlier at variable times due to convergence failure failure of the SCF or gradient module. However each trajectory died a short period after the Fe-CO dissociation. The survival rate of the trajectories as a function of time is shown in Figure $3 \mathrm{~b}$. All the analysis which are time dependent as done over these surviving trajectories only. Its noteworthy mentioning here that out of 110 trajectories only in 4 cases $\mathrm{Fe}(\mathrm{CO})_{5}$ remained intact after 600 fs and hence the 
photodissociation of $\mathrm{Fe}(\mathrm{CO})_{5}$ was captured properly. The non-adiabatic coupling between different states was handled by a local diabatization method using wavefunction overlap [42, 43]. The simulation was performed using a time step of $0.5 \mathrm{fs}$. The rest of the parameter used by SHARC was taken to be default values.

\section{Quantum chemistry}

All computations on TDDFT and NEVPT2 level were done with ORCA 4.2.0 [44], which also was used in an interface with SHARC [24, 32] for the TDDFT based excited state molecular dynamics simulations of the photodissociation of $\mathrm{Fe}(\mathrm{CO})_{5}$. DFT has been shown to accurately reproduce the thermodynamics for $\mathrm{CO}$ dissociation for $\mathrm{Fe}(\mathrm{CO})_{5}$ considering only the singlet manifold [45]. It is worth mentioning that TDDFT fails when multireference or near-degeneracy effects come into effect, but that occurs for the present system only after $\mathrm{CO}$ is released from the complex and the $\mathrm{Fe}(\mathrm{CO})_{4}$ fragment is formed which has closely lying $\mathrm{S}_{0}$ and $\mathrm{S}_{1}$ states. Thus TDDFT can be safely used to study the dissociation process itself. Using TDDFT, UV spectra and ESMD trajectories were computed with cam-B3LYP/def2TZVP functional [46, 47] with RIJCOSX to make the single point computations faster.

For the CASSCF/NEVPT2/CASPT2/def2TZVP calculations we employed a $(10,10)$ active space as employed by Pierloot and coworkers [14] and corroborates the findings of Daniel and coworkers [13]. CASSCF and the following perturbation theory computations were all carried out in ORCA 4.2.0 [44]. The NEVPT2 spectrum is reproduced in Figure S1.

\section{DATA AVAILABILITY}

The datasets generated and analyzed during the current study are available from the corresponding author on reasonable request.

[1] Wrighton, M. Photochemistry of metal carbonyls. Chemical Reviews 74, 401-430 (1974).

[2] Fuss, W., Trushin, S. \& Schmid, W. Ultrafast photochemistry of metal carbonyls. Research on Chemical Intermediates 27, 447-457 (2001). 
[3] Long, C. Photophysics of CO loss from simple metal carbonyl complexes. In Photophysics of Organometallics, 159-191 (Springer, 2009).

[4] Cho, H. et al. Electronic and molecular structure of the transient radical photocatalyst $\mathrm{Mn}(\mathrm{CO})_{5}$ and its parent compound $\mathrm{Mn}_{2}(\mathrm{CO})_{10}$. Inorganic Chemistry 55, 5895-5903 (2016).

[5] Schoenlein, R. et al. Recent advances in ultrafast x-ray sources. Philosophical Transaction of the Royal Society A 377, 20180384 (2019).

[6] Wernet, P. Chemical interactions and dynamics with femtosecond x-ray spectroscopy and the role of x-ray free-electron lasers. Philosophical Transaction of the Royal Society A $\mathbf{3 7 7}$, 20170464 (2019).

[7] Leadbeater, N. Enlightening organometallic chemistry: the photochemistry of $\mathrm{Fe}(\mathrm{CO})_{5}$ and the reaction chemistry of unsaturated iron carbonyl fragments. Coordination Chemistry Reviews 188, 35-70 (1999).

[8] Trushin, S., Fuss, W., Kompa, K. \& Schmid, W. Femtosecond dynamics of Fe(CO) 5 photodissociation at $267 \mathrm{~nm}$ studied by transient ionization. The Journal of Physical Chemistry A 104, 1997-2006 (2000).

[9] Ihee, H., Cao, J. \& Zewail, A. H. Ultrafast electron diffraction of transient $\mathrm{Fe}(\mathrm{CO})_{4}$ : Determination of molecular structure and reaction pathway. Angewandte Chemie International Edition 40, 1532-1536 (2001).

[10] Wernet, P. et al. Communication: Direct evidence for sequential dissociation of gas-phase $\mathrm{Fe}(\mathrm{CO})_{5}$ via a singlet pathway upon excitation at $266 \mathrm{~nm}$. The Journal of Chemical Physics 146, 211103 (2017).

[11] Leitner, T. et al. Time-resolved electron spectroscopy for chemical analysis of photodissociation: Photoelectron spectra of $\mathrm{Fe}(\mathrm{CO})_{5}, \mathrm{Fe}(\mathrm{CO})_{4}$ and $\mathrm{Fe}(\mathrm{CO})_{3}$. The Journal of Chemical Physics 149, 044307 (2018).

[12] Goumans, T. P. M. et al. Photodissociation of the phosphine-substituted transition metal carbonyl complexes $\mathrm{Cr}(\mathrm{CO})_{5} \mathrm{~L}$ and $\mathrm{Fe}(\mathrm{CO})_{4} \mathrm{~L}$ : A theoretical study. Journal of the American Chemical Society 125, 3558-3567 (2003).

[13] Rubner, O., Engel, V., Hachey, M. \& Daniel, C. A CASSCF/MR-CCI study of the excited states of $\mathrm{Fe}(\mathrm{CO})_{5}$. Chemical Physics Letters 302, 489-494 (1999).

[14] Persson, B. J., Roos, B. O. \& Pierloot, K. A theoretical study of the chemical bonding in $\mathrm{M}(\mathrm{CO})_{x}(\mathrm{M}=\mathrm{Cr}, \mathrm{Fe}$, and Ni). The Journal of Chemical Physics 101, 6810-6821 (1994). 
[15] Tsuchiya, T. \& Roos, B. A theoretical study of the spin-forbidden reaction $\mathrm{Fe}(\mathrm{CO})_{4}+\mathrm{CO} \rightarrow$ Fe $(\mathrm{CO})_{5}$. Molecular Physics 104, 1123-1131 (2006).

[16] Corriu, R. J., Lanneau, G. F. \& Chauhan, B. P. Photochemical reaction of 16-e metal species generated from $\mathrm{Fe}(\mathrm{CO})_{5}, \mathrm{Cr}(\mathrm{CO})_{6}$, or $\mathrm{RCpMn}(\mathrm{CO})_{3}(\mathrm{R}=\mathrm{H}, \mathrm{Me})$, with primary and secondary arylsilanes in the presence of internal or external electron donors: formation of functionally stabilized hydrosilanediyl-transition metal complexes. Organometallics 12, 2001-2003 (1993).

[17] Gandeepan, P. et al. 3d transition metals for $\mathrm{C}-\mathrm{H}$ activation. Chemical Reviews 119, 2192 $2452(2018)$.

[18] Lim, M., Jackson, T. A. \& Anfinrud, P. A. Binding of CO to myoglobin from a heme pocket docking site to form nearly linear Fe-CO. Science 269, 962-966 (1995).

[19] Wang, G., Ceylan, Y. S., Cundari, T. R. \& Dias, H. R. Heterobimetallic silver-iron complexes involving $\mathrm{Fe}(\mathrm{CO})_{5}$ ligands. Journal of the American Chemical Society 139, 14292-14301 (2017).

[20] Wang, G. et al. Heterobimetallic complexes featuring $\mathrm{Fe}(\mathrm{CO})_{5}$ as a ligand on gold. ChemistryA European Journal 23, 17222-17226 (2017).

[21] Cahoon, J. F., Sawyer, K. R., Schlegel, J. P. \& Harris, C. B. Determining transition-state geometries in liquids using 2D-IR. Science 319, 1820-1823 (2008).

[22] Wernet, P. et al. Orbital-specific mapping of the ligand exchange dynamics of $\mathrm{Fe}(\mathrm{CO})_{5}$ in solution. Nature 520, 78-81 (2015).

[23] Kunnus, K. et al. Identification of the dominant photochemical pathways and mechanistic insights to the ultrafast ligand exchange of $\mathrm{Fe}(\mathrm{CO})_{5}$ to $\mathrm{Fe}(\mathrm{CO})_{4} \mathrm{EtOH}$. Structural Dynamics 3, 043204 (2016).

[24] Mai, S., Marquetand, P. \& González, L. Nonadiabatic dynamics: The sharc approach. WIREs Computational Molecular Science 8, e1370 (2018).

[25] Liu, Y. et al. Spectroscopic and structural probing of excited-state molecular dynamics with time-resolved photoelectron spectroscopy and ultrafast electron diffraction. Physical Review $X$ 10, $021016(2020)$.

[26] Stankus, B. et al. Ultrafast x-ray scattering reveals vibrational coherence following rydberg excitation. Nature Chemistry 11, 716-721 (2019). 
[27] Cho, D. \& Mukamel, S. Stimulated x-ray raman imaging of conical intersections. The Journal of Physical Chemistry Letters 11, 33-39 (2019).

[28] Sánchez-Murcia, P. A., Perez, J. J. N., Plasser, F. \& González, L. Orbital-free photophysical descriptors to predict directional excitations in metal-based photosensitizers. Chemical Science (2020).

[29] Mai, S. \& González, L. Unconventional two-step spin relaxation dynamics of $\left[\operatorname{Re}(\mathrm{CO})_{3}(\mathrm{im})(\mathrm{phen})\right]^{+}$in aqueous solution. Chemical Science 10, 10405-10411 (2019).

[30] Crespo-Otero, R. \& Barbatti, M. $\mathrm{Cr}(\mathrm{CO})_{6}$ photochemistry: Semi-classical study of uv absorption spectral intensities and dynamics of photodissociation. The Journal of Chemical Physics 134, 164305 (2011).

[31] Supplementary information available at.... (2021).

[32] Richter, M., Marquetand, P., González-Vázquez, J., Sola, I. \& González, L. Sharc: ab initio molecular dynamics with surface hopping in the adiabatic representation including arbitrary couplings. Journal of Chemical Theory and Computation 7, 1253-1258 (2011).

[33] Rose, T. S., Rosker, M. J. \& Zewail, A. H. Femtosecond real-time probing of reactions. iv. the reactions of alkali halides. The Journal of Chemical Physics 91, 7415-7436 (1989).

[34] Virtanen, P. et al. Scipy 1.0: fundamental algorithms for scientific computing in python. Nature methods 17, 261-272 (2020).

[35] Dormand, J. R. \& Prince, P. J. A family of embedded Runge-Kutta formulae. Journal of computational and applied mathematics 6, 19-26 (1980).

[36] Branch, M. A., Coleman, T. F. \& Li, Y. A subspace, interior, and conjugate gradient method for large-scale bound-constrained minimization problems. SIAM Journal on Scientific Computing 21, 1-23 (1999).

[37] Abedi, M., Pápai, M., Mikkelsen, K. V., Henriksen, N. E. \& Møller, K. B. Mechanism of photoinduced dihydroazulene ring-opening reaction. The Journal of Physical Chemistry Letters 10, 3944-3949 (2019).

[38] Atkins, P. \& Overton, T. Shriver and Atkins' inorganic chemistry (Oxford University Press, USA, 2010).

[39] Kunnus, K. et al. Anti-stokes x-ray raman scattering for atom specific and excited state selective dynamics. New Journal of Physics 18, 103011 (2016). 
[40] Mai, S., Marquetand, P. \& González, L. A general method to describe intersystem crossing dynamics in trajectory surface hopping. International Journal of Quantum Chemistry 115, $1215-1231$ (2015).

[41] Barbatti, M. et al. The on-the-fly surface-hopping program system newton-x: Application to ab initio simulation of the nonadiabatic photodynamics of benchmark systems. Journal of Photochemistry and Photobiology A: Chemistry 190, 228-240 (2007).

[42] Plasser, F. et al. Surface hopping dynamics using a locally diabatic formalism: Charge transfer in the ethylene dimer cation and excited state dynamics in the 2-pyridone dimer. The Journal of chemical physics 137, 22A514 (2012).

[43] Granucci, G., Persico, M. \& Toniolo, A. Direct semiclassical simulation of photochemical processes with semiempirical wave functions. The Journal of Chemical Physics 114, 1060810615 (2001).

[44] Neese, F., Wennmohs, F., Becker, U. \& Riplinger, C. The orca quantum chemistry program package. The Journal of Chemical Physics 152, 224108 (2020).

[45] Gonzalez-Blanco, O. \& Branchadell, V. Density functional study of the Fe-CO bond dissociation energies of $\mathrm{Fe}(\mathrm{CO})_{5}$. The Journal of Chemical Physics 110, 778-783 (1999).

[46] Yanai, T., Tew, D. P. \& Handy, N. C. A new hybrid exchange-correlation functional using the Coulomb-attenuating method (CAM-B3LYP). Chemical Physics Letters 393, 51-57 (2004).

[47] Weigend, F. \& Ahlrichs, R. Balanced basis sets of split valence, triple zeta valence and quadruple zeta valence quality for $\mathrm{H}$ to $\mathrm{Rn}$ : Design and assessment of accuracy. Physical Chemistry Chemical Physics 7, 3297-3305 (2005). 

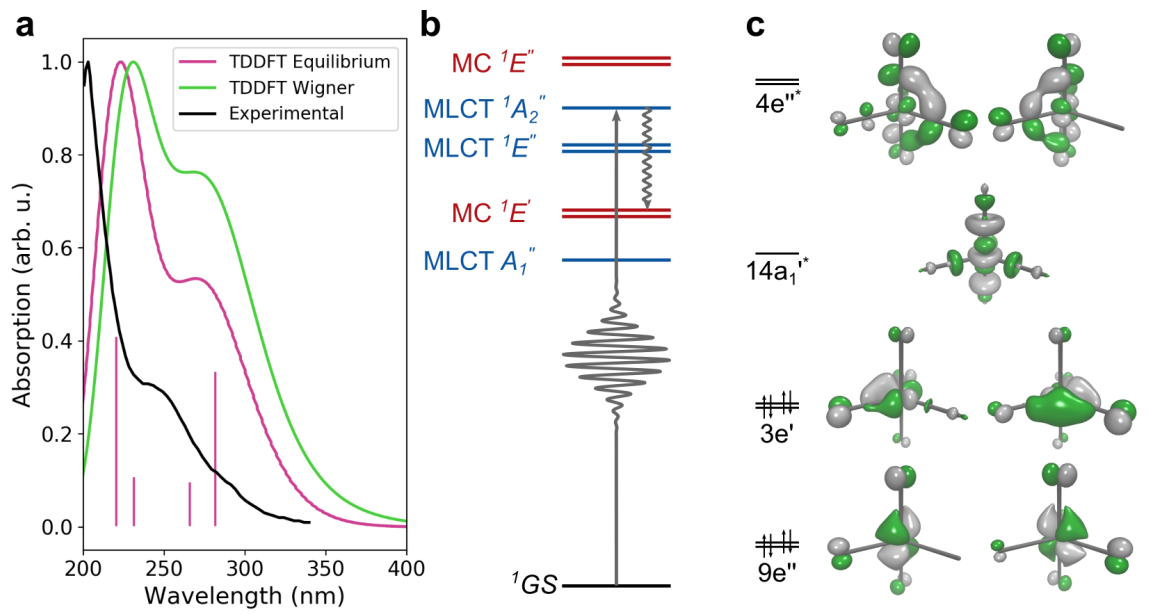

FIG. 1. Photoexcitation, energy levels and frontier molecular orbitals of ironpentacarbonyl $\mathbf{F e}(\mathbf{C O})_{5}$. a The TDDFT simulated UV spectra for the equilibrium geometry (pink curve), Wigner distribution (green curve) and the experimental gas phase UV spectrum (black curve) are shown here.

b Level diagram of electronic states involved in the photoexcitation and in the subsequent dissociation process. The character of metal-to-ligand charge-transfer (MLCT) and metal-centered (MC) states is denoted by red and blue color, respectively. c Frontier molecular orbitals involved in the photodissociation of ironpentacarbonyl, with specifications of of orbital symmetries and ground state occupation. 

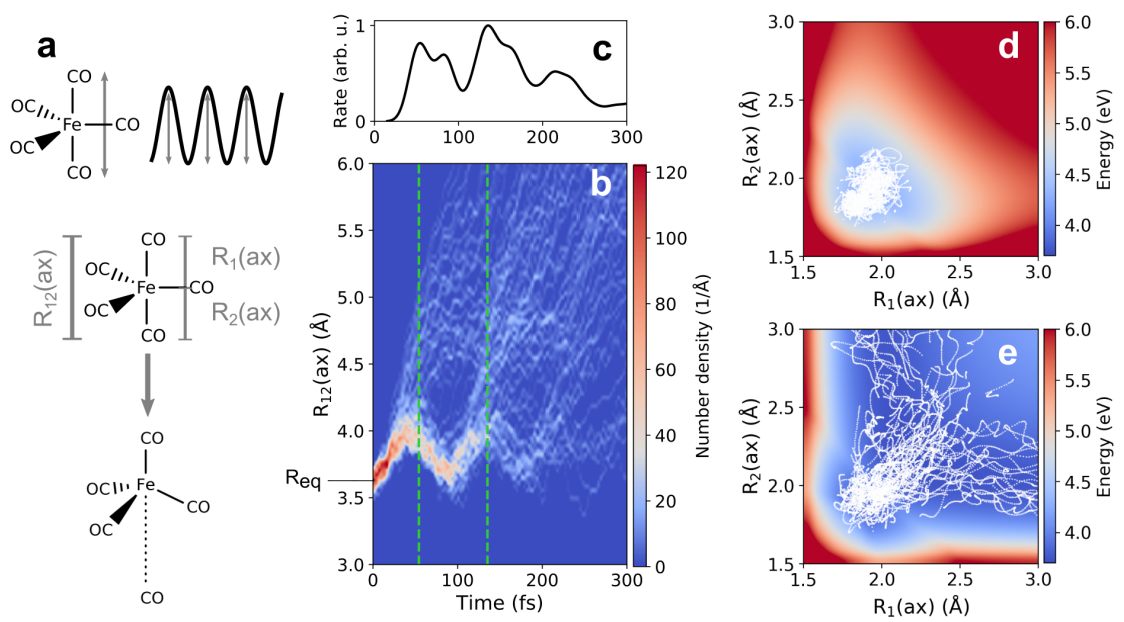

FIG. 2. Release of axial CO from photoexcited Fe(CO) $)_{5}$ synchronous with the symmetric stretch mode in axial Fe-C bonds. a Schematic diagram for definition and relevance of the $\mathrm{R}_{12}(\mathrm{ax})$ coordinate in axial $\mathrm{CO}$ dissociation. $\mathbf{b}$ Plot of distribution of $\mathrm{R}_{12}(\mathrm{ax})$ as a function of time for the 94 trajectories yielding axial $\mathrm{CO}$ release. c Rate of axial $\mathrm{CO}$ release as a function of time (obtained from the dissociation times $\tau_{\text {dissoc }}$ ) showing clearly visible periodic bursts $\sim 90 \mathrm{fs}$ apart. The rate curved is obtained by applying a smoothing Gaussian convolution of the distribution of $\tau_{\text {dissoc }}$ with a full-width half-maximum of $10 \mathrm{fs}$. $\mathbf{d}$ and e Two-dimensional cuts in the potential energy surfaces (PES) of $\mathrm{S}_{6}(\mathrm{top})$ and $\mathrm{S}_{3}$ (bottom) states obtained by TDDFT scans along the axial Fe-C $\mathrm{R}_{1}(\mathrm{ax})$ vs $\mathrm{R}_{2}(\mathrm{ax})$ distances. The white scatter overlaid on the PES shows segments populating these states in the trajectories in $\mathbf{b}$. 


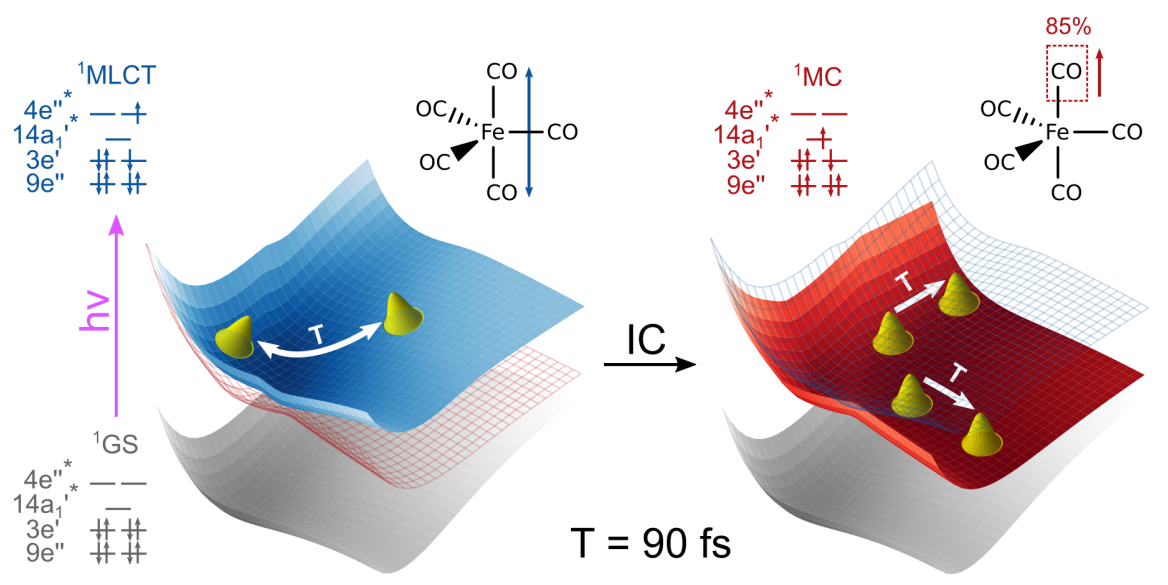

Scheme 1. Schematic representation of the oscillation in the MLCT state and the periodic release of predominantly axial CO ligands in a MC state. Orbital configurations of specific LMCT and MC states are given. The oscillation of the two axial COs in the bound MLCT state occurs with a period of $\sim 90 \mathrm{fs}$ which leads to the periodic crossover to the dissociative MC states at the same frequency. These lead to periodic bursts of $\mathrm{CO}$ dissociation having a time lag of $90 \mathrm{fs}$. 

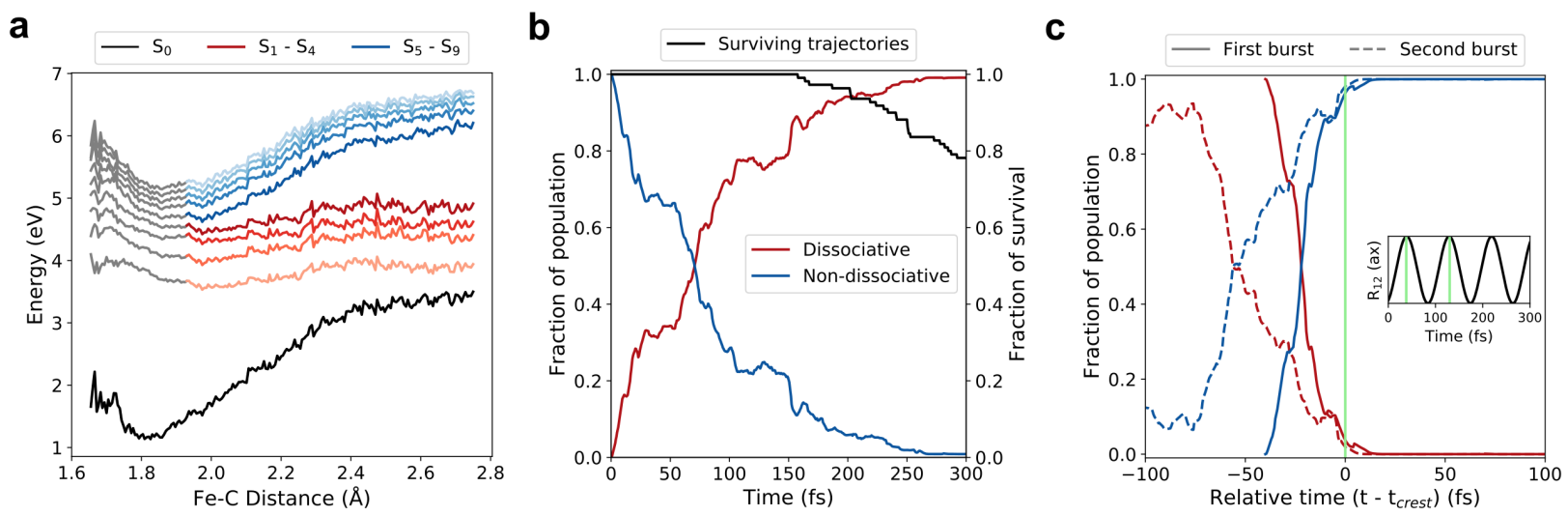

FIG. 3. Electronic population dynamics for the photodissociation of $\mathrm{Fe}(\mathrm{CO})_{5}$. a The potential energy of all the states at each time step for the singly dissociative trajectories (110) were averaged and plotted as a function of Fe-C distance, corresponding to the released $\mathrm{CO}$. At long Fe-C distance, non-dissociative and dissociative states are colored blue and red, respectively. b Population dynamics of non-dissociative, ie. $\mathrm{S}_{i}(\mathrm{i}=5-9)$, and dissociative, ie. $\mathrm{S}_{j}(\mathrm{i}=1-4)$, states averaged over the 110 single dissociation trajectories. The black line represents the fraction of trajectories that survives as function of time. c Normalized population dynamics for the two prominent bursts in subset of trajectories with axial dissociation from Figure 2 presented on a relative time axis. The relative time 0 fs for first and second burst matches the corresponding crests of the oscillation of $R_{12}(a x)$, respectively. The inset shows the schematic $R_{12}(a x)$ oscillation and the green lines indicate the positions of $t_{\text {crest }}$ for first and second burst of axial CO ligands. 


\section{a}
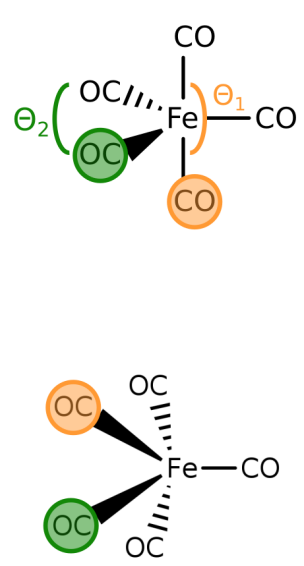

b

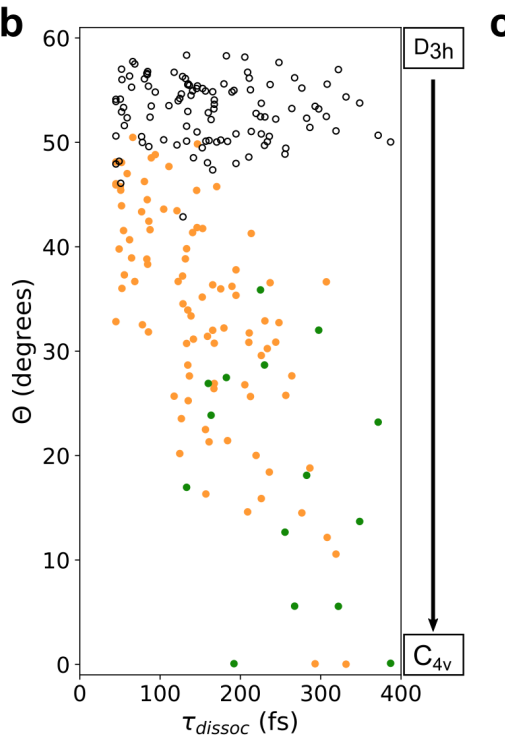

C

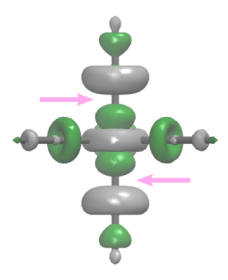

$D_{3 h}$ side-view

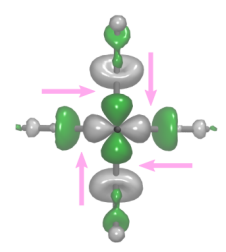

$\mathrm{C}_{4 \mathrm{v}}$ top-view

FIG. 4. Correlation of angular distortion, from $\mathbf{D}_{3 h}$ geometry to near $\mathbf{C}_{4 v}$ geometry as measured by decrease in $\Theta$, and time taken for $\mathrm{CO}$ release. a Definition of angular distortion parameter $\Theta$ involving transition from $\mathrm{D}_{3 h}$ geometry to near $\mathrm{C}_{4 v}$ geometry; followed by release of $\mathrm{CO}$. The green and orange spheres denote that the axial and equatorial ligands become equivalent upon angular distortion to the nearly $\mathrm{C}_{4 v}$ geometry. $\mathbf{b}$ Scatter plot of the smallest value of $\Theta$, before $\tau_{d i s s o c}$, versus $\tau_{\text {dissoc }}$ for all 110 singly dissociative trajectories. The orange and green solid dots represent release of axial and equatorial CO ligands, respectively. The hollow black dots show the initial distribution of $\Theta$ obtained from the Wigner distribution. c The excited singly occupied molecular orbital involved in the the dissociative MC states at ground state $\mathrm{D}_{3 h}$ geometry (top) and in the near $\mathrm{C}_{4 v}$ geometry (bottom) which is also the optimized geometry for the $\mathrm{S}_{5}$ state. The pink arrows for both the cases indicate regions of antibonding overlap which effectuates the CO dissociation. 